\title{
Intracranial Hemangiopericytoma - A Case Report
}

\author{
Gilson Gabriel Viana Veloso ${ }^{1}$, Raul de Almeida Dutra ${ }^{2}$, Letícia de Almeida \\ Dutra $^{4}$, Pedro Teixeira Meireles ${ }^{4}$, Douglas Reis Abdalla ${ }^{4,5}$, Luiz Carlos Furtado \\ de Almeida Júnior ${ }^{3,4}$
}

${ }^{1}$ Clinic Oncologist, Hospital Santa Casa de Belo Horizonte, Brazil. ${ }^{2}$ Clinic Oncologist, Hospital Doutor Hélio Angotti, Brazil. ${ }^{3}$ Surgical Oncology, Hospital Doutor Hélio Angotti, Brazil. ${ }^{4}$ Medical Student, Universidade de Uberaba, Brazil. ${ }^{5}$ Health Sciences, Faculdade de Talentos Humanos, Brazil.

\begin{abstract}
Hemangiopericytoma is a rare mesenchymal tumor, considered as a subtype of soft tissue sarcomas with an incidence of 3.77/10.000.000 cases. Intracranial hemangiopericytomas account for less than $1 \%$ of all primary tumors of the central nervous system. Due to the lack of data available regarding this disease, national and international research provides information derived from retrospective case series and phase I and phase II studies. This is a case report of a young adult diagnosed with intracranial hemangiopericytoma in 2012 with a history of multiple recurrences and therapeutic approaches, assisted in a public health center in southeastern Brazil. Among the treatment options given to the patient, there are surgery, radiotherapy, and systemic chemotherapy (including alkylating and anthracyclines agents). Systemic therapy usually is offered after multiple recurrences or in a metastatic setting; although it increases progression-free survival, there is not enough data on objective response rate and does not yield increased overall survival.
\end{abstract}

Keywords: Hemangiopericytoma-Sarcoma- Oncology- Tumor

Asian Pac J Cancer Care, 5 (4), 351-354

\section{Introduction}

Cancer is a non-transmissible disease considered as the second leading cause of death worldwide (approximately $21 \%$ ) [1]. For 2019, 600 thousand new cancer cases were expected in Brazil [1].

Hemangiopericytomas are rare mesenchymal tumors originating from pericytes' proliferation and characterized by a distinguished biological and clinical behavior. They account for less than $1 \%$ of intracranial tumors [2], with an incidence of $3.77 / 10.000 .000$ [3] and are classified as soft tissue sarcomas and usually affect individuals between 20 and 70 years of age [4].

Incidence and prevalence data of malignant neoplasms in Brazil are usually presented biannually by José Alencar Gomes da Silva National Cancer Institute (INCA) [5]. However, due to underreported cases and a heterogenous distribution of cancer types among Brazilian regions, an accurate data regarding Brazil's cancer incidence and prevalence still is not available. Corroborating with these
Submission Date: 07/07/2020Ａcceptance Date: 11/07/2020 facts, INCA's Estimates 2018 book does not contain data on the prevalence and incidence of soft tissue tumors.

Therefore, this study aims to report a case of a young adult with intracranial hemangiopericytoma, a rare malignant neoplasm, with fewer descriptions on national and international literature, since its' treatment is based on results of case reports like this one.

\section{Case Report}

This case report, which was accepted on the ethics committee under the registration number: CAAE 33375120.6.0000.9028, was about a patient of 22-year-old male with prior tumor excision in the left hemiface (with orbital invasion) in 2012 and an anatomopathological result of fusocellular neoplasia. During this period, he was not assessed by a clinical oncologist and no additional treatment was performed.

About 5 years later, in 2017, he presented with an

Corresponding Author:

Dr. Douglas Reis Abdalla

${ }^{4}$ Medical Student, Universidade de Uberaba, Brazil. ${ }^{5}$ Health Sciences, Faculdade de Talentos Humanos, Brazil.

Email: drabdalla@facthus.edu.br 
episode of severe headache and vomiting, refractory to medication. A new investigation was initiated, which detected an extensive tumor recurrence. Head computed tomography (Figure 1) scan showed a lesion of $7.9 \times 8.4$ x $9.3 \mathrm{~cm}$, located in the middle fossa and infratemporal fossa on the left with contralateral midline deviation. After diagnosis, he underwent tumor embolization and partial excision in July 2017; the anatomopathological report came as a mesenchymal neoplasia and the hypotheses of sarcoma and hemangiopericytoma/malignant solitary fibrous tumor were questioned. An immunohistochemistry of the first surgical specimen (2012) was requested, which came positively for the STAT6 markers, favoring the diagnosis of hemangiopericytoma; other markers included ERG, p63, desmin, AML, HMB45, CD34, S100, AE1AE3, which were all negative.

In August 2017, he started external beam radiotherapy (60 Gy/30 fractions) and chemotherapy with doxorubicin at a dose of $50 \mathrm{mg} / \mathrm{m}^{2}$ every 21 days (total of 9 cycles maximum dosage).

Clinical assessment during and after treatment indicated a partial response, with a residual tumor of 4.9 x 6.7 x $4.5 \mathrm{~cm}$ (contained by the dura mater), which was centered on the left sphenoid bone and extended to the middle cranial fossa, without invasion of the cerebral parenchyma or mass effect on the temporal lobe.

At the end of chemotherapy, he remained in clinical follow-up with Oncology and Neurology. After 18 months, he presented with episodes of massive epistaxis and maxillary sinusitis. A brain magnetic resonance imaging identified a bulky expansive and infiltrative lesion roughly measuring $5.7 \times 4.5 \times 4.8 \mathrm{~cm}$, centered on the left hemiface, predominantly in nasal meatus on this side, with invasion of the maxillary sinus, nasopharynx, middle cranial fossa, cavernous sinus and turkish saddle. Tumor invaded adjacent bone structures with apparent destruction on the left side (nasal septum, septa of ethmoidal cells and left maxillary sinus walls); additionally, it was involving the carotid arteries at the intracavernous portions.

Subject underwent a new surgical approach by both Otorhinolaryngology and Head and Neck surgical teams. The surgery consisted in a left subtotal maxillectomy, left ethmoidectomy with resection of the middle concha

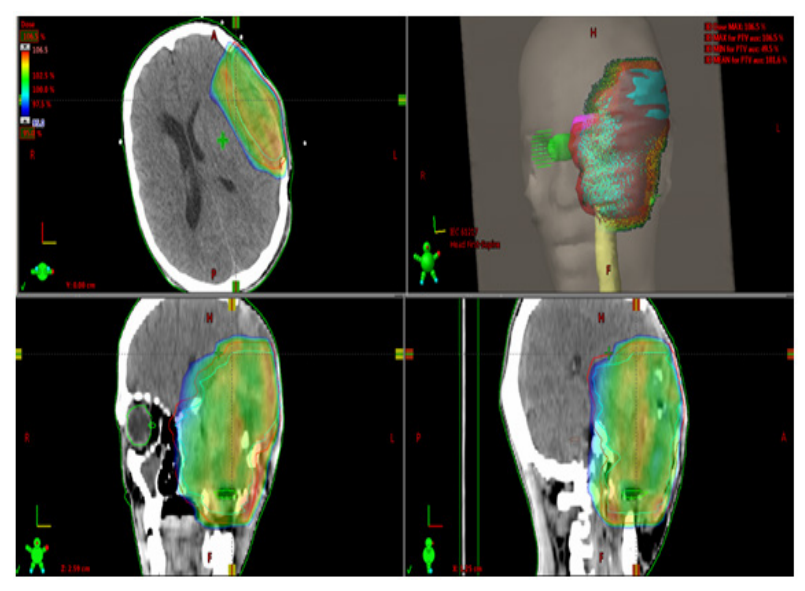

Figure 1. 2017 RT Planning Image and papyraceous lamina and spheneoidectomy for tumor resection by means of mediofacial Hemidegloving access. An exploration of the left pterygopalatine and infratemporal fossa was performed, as well as the exposure of the internal carotid artery, which was preserved. There was no major bleeding or surgical complications. After recovery, patient was again referred to consolidation radiotherapy and the resected piece was sent to the anatomopathological.

Although he was referred to consolidation radiotherapy, for personal reasons, patient did not attend the appointed consults or the scheduled radiotherapy sessions. He remained on clinical follow-up. The latest head and facial computed tomography scan (done 4 months after surgery), Figure 2, showed a hypodense, irregular amorphous area partially delimited, located posterolateral to the left orbit, measuring approximately $3.6 \times 3.1 \times 2.7 \mathrm{~cm}$, with an estimated volume of $15.6 \mathrm{~cm}^{3}$. Such lesion invades the left orbit and touches the corresponding eyeball and lateral rectus muscle, without a well-defined cleavage plane with these structures.

On the latest clinical appointment (February 2020), patient was considered clinically stable, despite having left amaurosis and being partially dependent for daily-life activities. Pain management included methadone (10mg 3 times a day) and amitriptyline (50mg per day).

\section{Discussion}

The first description of hemangiopericytoma occurred in 1942, when Stout and Murray identified tumors that derived from Zimmermann's pericytes [6]. The first intracranial case was published only in 1954 by Begg and Garret (1954) [7].

The solitary fibrous tumor and the hemangiopericytoma share similar histopathological characteristics and are now classified as a single biological entity. Both tumors have a NAB2-STAT6 fusion, regardless of the anatomical location (pleura, meninges or soft tissues) [8]. The fusion of the NAB2 and STAT6 genes generates nuclear expression of STAT6 (detected in immunohistochemistry) 9. This characteristic is fundamental for a correct diagnosis of this pathology, being the reason why is analyzed in all suspected cases. In a normal genome, NAB2 and STAT6 are adjacent genes on chromosome 12q13 and are transcribed in opposite directions. In a fibrous tumor, however, chromosomal inversion occurs, fusing these two

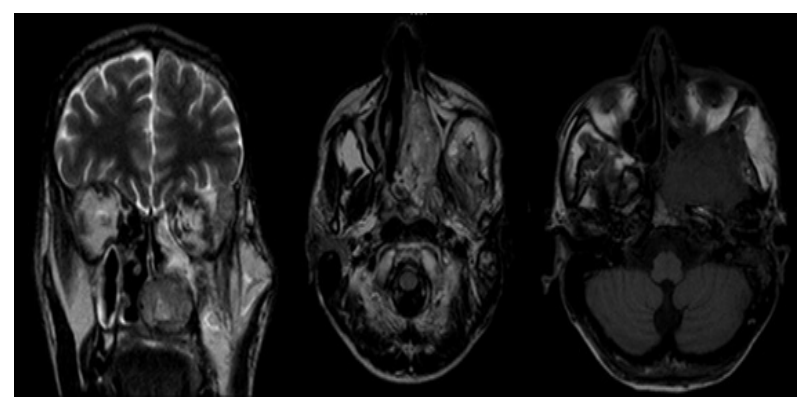

Figure 2. Skull MRI of Tumor Recurrence in 2019 
genes in the same direction of transcription [8]. From this fusion, stimulation of several growth factors and greater expression of tyrosine kinase receptors occurs, inducing tumor growth.

The most common sites of involvement of these tumors include lower limbs, retroperitoneum/pelvis, lung/pleura and meninges [9]. The symptoms of hemangiopericytoma include headache, dizziness, vomiting, asthenia and blurred vision. Additional signs may appear on physical examination, such as papilledema, hemiparesis, ataxia and other focal neurological deficits [10].

Due to its rarity, there is no standard treatment [10]. Published data on hemangiopericytoma are scarce and the ones currently available are based only on case reports and retrospective analyzes regarding treatment and tumor biological behavior.

Primary complete surgical resection is the consensual treatment for localized disease; it is de only treatment modality related to an increased overall survival [10] reaching a $100 \%$ five-year overall survival, independently of tumor primary site [11] and up to an $89 \%$ ten-year overall survival [12]. However, intracranial tumors have higher rates of local and distant recurrence [13] - approximately $43 \%$ and $15 \%$, respectively [14]. Preoperative tumor embolization may be considered as a measure of reducing the risk of bleeding during surgery, since these types of cancer have a large vascularization [10].

Regarding adjuvant radiotherapy, the retrospective analysis published that described 17 patients with intracranial hemangiopericytoma, treated between 1995 and 2014 [15]. All patients underwent initial surgical treatment and 9 required re-approaches; no patient had distant metastasis. Adjuvant radiotherapy was administered to 16 patients regardless of the resection pattern (total or partial) with a median of $57.57 \mathrm{~Gy} / 33$ fractions. Among those analyzed, no patient received adjuvant chemotherapy. The median progression-free survival was 51 months and the median overall survival was not reached.

Another study by Patel et al (2017) carried out a retrospective analysis of 20 patients with hemangiopericytomas with a mean age of 45.6 years, assisted between 1985 and 2014 demonstrated that headache, visual disturbances and seizures were among the most common reported symptoms. Treatment focused mainly on surgical resection followed by adjuvant radiotherapy. The use of systemic chemotherapy was considered in cases of multiple recurrences. It was also pointed out that despite advances in surgical and radiotherapy techniques, these tumors continue to have high recurrence rates and require adequate followup [13].

Management of recurrent and metastatic disease is not well established and that a complete surgical resection does not eliminate the risk of local tumor recurrence (commonly in the same surgical bed) [10].

From all studied and currently available treatment options for hemangiopericytomas, there is monoclonal antibodies, mTOR inhibitors, tyrosine kinase inhibitors, target therapies and the use of cytotoxic chemotherapy.
However, most published data are derived from case series, phase 1 and phase 2 studies, making it difficult for Oncologists to establish a standard therapy. In addition, part of the studies included patients with various types of soft tissue sarcomas and not solely hemangiopericytomas.

In this context, the progression-free survival and overall survival rates reported in these studies are low, such as the use of temozolamide and bevacizumab (median 10.8 months and 24.3 months, respectively) [16]. For sunitinib, the median duration of treatment was 6 months [17] and for sorafenib, the medians for progression-free survival and overall survival were 3 and 17 months, respectively [18]. Pazopanib produced similar results, with a median progression-free survival of 4.7 months and median overall survival of 13.3 months [19]. Regarding the use of mTOR and IGF-1 inhibitors (analyzed after at least 2 lines of treatment), the results were disappointing [20]. It is necessary to keep in mind that there are no comparative efficacy data of those drugs in relation to the systemic chemotherapy regimens currently used such as anthracyclines.

Regarding chemotherapy, a retrospective study by Park et al (2013), showed the effectiveness in 21 patients assisted at the MD Anderson Cancer Center between 1994 and 2007. All subjects had measurable and advanced disease (unresectable, metastatic or potentially resectable) as a prerequisite for treatment. Among the chemotherapy regimens used, those based on doxorubicin were the majority $(60 \%)$. Others included gemcitabine with docetaxel and paclitaxel. It is worth mentioning that 4 patients received more than 01 treatment regimen. Best objective response rate was stable disease and there was no complete or partial response. Median progression-free survival was 4.6 months and median overall survival was 10.3 years since diagnosis.

\section{Final Considerations}

Hemangiopericytomas of the central nervous system are rare neoplasms with unpredictable behavior. In the absence of randomized clinical trials and large-scale studies, the only source of information on its natural history and clinical management is available from isolated case series.

In this context and based on what is currently published, surgical treatment associated with radiotherapy is valued to achieve better therapeutic efficacy. Conventional chemotherapy is effective in stabilizing locally advanced or metastatic disease and despite having minimal response rates (partial or complete clinical response), the clinical benefit achieved by reducing symptoms and stabilizing the disease justify its use in medical practice.

\section{References}

1. Brasil. Instituto Nacional de Câncer José Alencar Gomes da Silva. Coordenação de Prevenção e Vigilância. Estimativa 2018: incidência de câncer no Brasil / Instituto Nacional de Câncer José Alencar Gomes da Silva. Coordenação de Prevenção e Vigilância. - Rio de Janeiro: INCA. 2017;:128p. 2. Seo I, Kim Y, Kim H, Kim J, Lee M. Hemangiopericytoma 
of the Posterior Fossa: A Case Report and Review of the Literature. Brain Tumor Research and Treatment. 2013;1(2):95. https://doi.org/10.14791/btrt.2013.1.2.95

3. Kinslow CJ, Bruce SS, Rae AI, Sheth SA, McKhann GM, Sisti MB, Bruce JN, Sonabend AM, Wang TJC. Solitary-fibrous tumor/hemangiopericytoma of the central nervous system: a population-based study. Journal of Neuro-Oncology. 2018 02 09;138(1):173-182. https://doi.org/10.1007/s11060-0182787-7

4. Cheng KP, Won W, Hashim S, Mun KS. Hemangiopericytoma 11 years later: delayed recurrence of a rare soft tissue sarcoma. Journal of Thoracic Disease. 2017 09;9(9):E752-E756. https://doi.org/10.21037/jtd.2017.08.74

5. Brasil. [Internet] Instituto Nacional de Câncer José Alencar Gomes da Silva (INCA). Estatísticas do câncer; [atualizado em 2019 Ago 01; citado em 2019 Out 31]. Disponível em: https://www.inca.gov.br/numeros-decancer..

6. STOUT AP, MURRAY MR. HEMANGIOPERICYTOMAA VASCULAR TUMOR FEATURING ZIMMERMANN'S PERICYTES. Annals of Surgery. 1942 07;116(1):26-33. https://doi.org/10.1097/00000658-194207000-00004

7. Begg CF, Garret R. Hemangiopericytoma occurring in the meninges.Case report. Cancer. 1954 05;7(3):602-606. https://doi.org/10.1002/1097-0142(195405)7:3<602::aidcncr2820070319>3.0.co;2-a

8. Devita, Hellman, and Rosenberg's cancer: principles \& practice of oncology / editors, Vincent T. DeVita, Jr., Theodore S. Lawrence, Steven A. Rosenberg. Description: 11th edition. Philadelphia: Wolters Kluwer, [2019]..

9. Park MS, Ravi V, Conley A, Patel SR, Trent JC, Lev DC, Lazar AJ, Wang W, Benjamin RS, Araujo DM. The role of chemotherapy in advanced solitary fibrous tumors: a retrospective analysis. Clinical Sarcoma Research. 2013;3(1):7. https://doi.org/10.1186/2045-3329-3-7

10. Ciliberti MP, D’Agostino R, Gabrieli L, Nikolaou A, Sardaro A. The radiation therapy options of intracranial hemangiopericytoma: An overview and update on a rare vascular mesenchymal tumor. Oncology Reviews. 201807 10;. https://doi.org/10.4081/oncol.2018.354

11. Espat NJ, Lewis JJ, Leung D, Woodruff JM, Antonescu CR, Shia J, Brennan MF. Conventional hemangiopericytoma. Cancer. 2002 Oct 03;95(8):1746-1751. https://doi. org/10.1002/cncr.10867

12. Magdeleinat P, Alifano M, Petino A, Le Rochais J, Dulmet E, Galateau F, Icard P, Regnard J. Solitary fibrous tumors of the pleura: clinical characteristics, surgical treatment and outcome. European Journal of Cardio-Thoracic Surgery. 2002 06;21(6):1087-1093. https://doi.org/10.1016/s10107940(02)00099-4

13. Patel A, Flores B, Ban V, Hatanpaa K, Mickey B, Barnett S. Intracranial Hemangiopericytomas: Recurrence, Metastasis, and Radiotherapy. Journal of Neurological Surgery Part B: Skull Base. 201703 01;78(04):324-330. https://doi. org/10.1055/s-0037-1599073

14. Zanasi Junior S, Lozano P, de Sá V, Pereira Filho G, Heinke T. Hemangiopericitoma de órbita. Rev Bras Cir Plást. 2012;27(3):487-9.

15. Kim Y, Park J, Kim Y, Jeun S. Treatment Strategy of Intracranial Hemangiopericytoma. Brain Tumor Research and Treatment. 2015;3(2):68. https://doi.org/10.14791/ btrt.2015.3.2.68

16. Park MS, Patel SR, Ludwig JA, Trent JC, Conrad CA, Lazar AJ, Wang W, Boonsirikamchai P, Choi H, Wang X, Benjamin RS, Araujo DM. Activity of temozolomide and bevacizumab in the treatment of locally advanced, recurrent, and metastatic hemangiopericytoma and malignant solitary fibrous tumor. Cancer. 201104 08;117(21):4939-4947. https://doi.org/10.1002/cncr.26098

17. Stacchiotti S, Negri T, Palassini E, Conca E, Gronchi A, Morosi C, Messina A, Pastorino U, Pierotti MA, Casali PG, Pilotti S. Sunitinib Malate and Figitumumab in Solitary Fibrous Tumor: Patterns and Molecular Bases of Tumor Response. Molecular Cancer Therapeutics. 2010 05 01;9(5):1286-1297. https://doi.org/10.1158/1535-7163. mct-09-1205

18. von Mehren M, Rankin C, Goldblum JR, Demetri GD, Bramwell V, Ryan CW, Borden E. Phase 2 Southwest Oncology Group-directed intergroup trial (S0505) of sorafenib in advanced soft tissue sarcomas. Cancer. 2011 07 12;118(3):770-776. https://doi.org/10.1002/cncr.26334

19. Maruzzo M, Martin-Liberal J, Messiou C, Miah A, Thway K, Alvarado R, Judson I, Benson C. Pazopanib as first line treatment for solitary fibrous tumours: the Royal Marsden Hospital experience. Clinical Sarcoma Research. 2015;5(1):5. https://doi.org/10.1186/s13569-015-0022-2

20. Quek R, Wang Q, Morgan JA, Shapiro GI, Butrynski JE, Ramaiya N, Huftalen T, Jederlinic N, Manola J, Wagner AJ, Demetri GD, George S. Combination mTOR and IGF-1R Inhibition: Phase I Trial of Everolimus and Figitumumab in Patients with Advanced Sarcomas and Other Solid Tumors. Clinical Cancer Research. 2010 Dec 22;17(4):871-879. https://doi.org/10.1158/1078-0432.ccr-10-2621

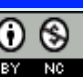

This work is licensed under a Creative Commons AttributionNon Commercial 4.0 International License. 